\title{
Analysis of Emergency Department Visits for Motor Vehicle Injuries in Utah, 2016
}

\author{
Akanksha Acharya \\ Bureau of Epidemiology, Utah Department of Health, Salt Lake City, Utah, United States \\ Objective
}

To describe the characteristics of emergency department (ED) visits for motor vehicle injuries in Utah using 2016 syndromic surveillance data.

\section{Introduction}

Motor vehicle injury is the leading cause of death in injury category in the United States. In 2016, motor vehicle crashes were one of the main causes of death resulting from injury ( 8.8 per 100,000 population) in Utah. Motor vehicle crashes can lead to physical and economic consequences that impact the lives of individuals and their families. In addition, the treatment of injuries places an enormous burden on hospital Emergency Departments (EDs). Currently; there are no data sources other than syndromic data in the Utah Department of Health to monitor ED visits due to motor vehicle injuries in real time.

\section{Methods}

Utah participates in the National Syndromic Surveillance Program (NSSP) to which all hospitals in the state submit ED visit data via the Electronic Surveillance System for the Early Notification of Community-based Epidemics (ESSENCE). ESSENCE was used to analyze 2016 ED visit data. Total population data were obtained from Utah population estimates. Data from 2017 was not included due to major system changes at a major healthcare system that interrupted data feeds resulting in lower than expected data volume.

Motor vehicle injury is defined by existing subsyndrome definition in the Centers for Disease Control and Prevention ESSENCE system. All ED visit data were analyzed by querying key terms in the chief complaint field including any mention of: vehicle, wheeler, motorcycle, motor scooter, motor cycle, motor cross, truck, motorbike etc. Exclusion terms included any mention of: car dealership, hit head and car door. Ages were divided into seven groups for data distribution and comparison: $0-17,18-24,25-34,35-44,45-54,55-64$ and $\geq 65$ years.

\section{Results}

In 2016, a total of 28,472 ED visits ( $2 \%$ of total visits) were identified using the motor vehicle injury query. The ED visit rate for motor vehicle injuries was highest among persons aged 18-24 years (1,682 per 100,000 population). Rates continued to decline with increasing age after 18-24 years. The rate of females visiting the ED was higher than males (1,040 versus 826 per 100,000 population respectively; $p<0.01)$ (Figure 1$)$. The majority of injuries $(11722(52 \%)$ ) were reported between 10:00 a.m. and 5:59 p.m. Injuries were highest August-September (5913(22\%)).

\section{Conclusions}

Syndromic data is a robust source of data for analyzing ED visits due to motor vehicle injuries in real time, and providing information to injury prevention programs for targeting interventions. Our data suggest an increased risk of visiting an ED due to motor vehicle injuries by age group (18-24 year olds), sex (females), month (August-September), and time (10:00 a.m. to 5:59 p.m.). These results do not include visits with incomplete or incorrectly coded chief complaints or discharge codes, patients of motor vehicle injuries who do not present to the ED, or not classified as 'emergency' patient class. 
Figure 1: Emergency Department Visit Rate for Motor Vehicle Injuries per 100,000 population by age and Sex, Utah 2016

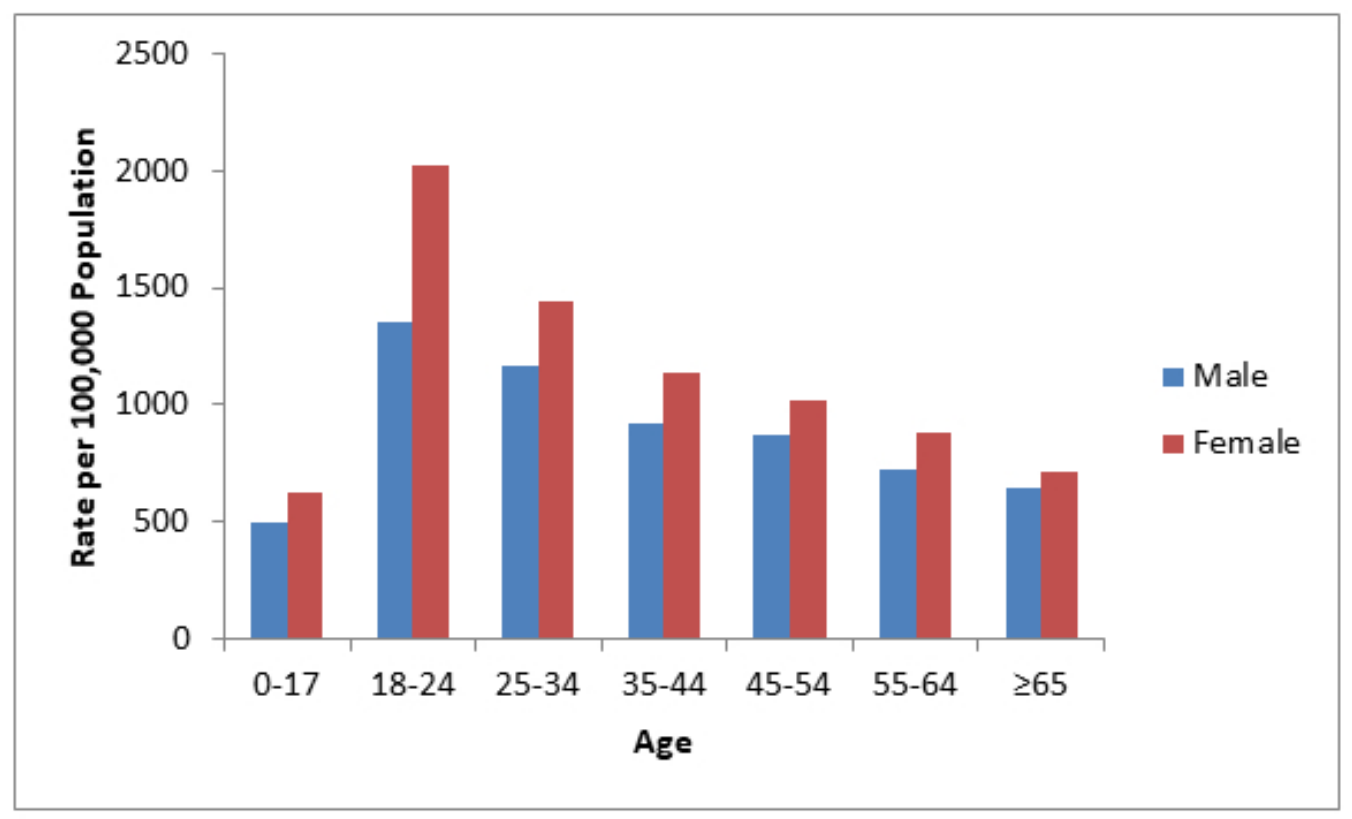

ISDS Annual Conference Proceedings 2019. This is an Open Access article distributed under the terms of the Creative Commons AttributionNoncommercial 3.0 Unported License (http://creativecommons.org/licenses/by-nc/3.0/), permitting all non-commercial use, distribution, and reproduction in any medium, provided the original work is properly cited. 